\title{
A comparison of forward and backward procedures for the acquisition of response chains in pigeons
}

\author{
RICHARD PISACRETA \\ Ferris State College, Big Rapids, Michigan 49307
}

\begin{abstract}
Four pigeons each learned two separate four-link response chains on a nine-key intelligence panel. One chain was acquired with forward chaining, and the other was learned with backward chaining. The first chain employed four colors as the discriminative stimuli $\left(\mathrm{S}^{\mathrm{D}} \mathbf{s}\right)$, and the second presented four forms. Completion of each chain produced 3 sec access to mixed grain, a 3-sec intertrial interval (ITI), and the next trial. Incorrect responses produced a 3-sec timeout (TO). During ITI and TO, all illumination in the experimental chamber was eliminated. All four birds made fewer errors in whichever chaining procedure they learned first, forward or backward.
\end{abstract}

When two or more responses, repetitive or emitted as different operants, must occur in a particular sequence in order to produce reinforcement, the behavior is said to be maintained as a behavioral chain. Most textbooks stress that chains of behavior are best established in a backward direction. That is, the last member of a chain is established first, followed by the acquisition, sequentially, of earlier links in the chain.

"Strengthen the members that occur nearest primary reinforcement first; that is build the chain from back to front" (Millenson, 1967, p. 260). "In order to establish a heterogeneous chain of responses, an experimenter begins with the last member of the chain and works backward" (Williams, 1973, p. 86). "The law of chaining. The response of one reflex may constitute or produce the eliciting or discrimination stimulus of another" (Skinner, 1938, p. 32). "The important rule in establishing chains is to start with the last response-the one that is rewarded with primary reinforcement" (Rachlin, 1976, p. 121).

Actual demonstrations of complex chains established by backward chaining have been few (e.g., Pierrel \& Sherman, 1963). However, several procedures well represented in the literature have employed forward chaining. The matching-to-sample paradigm typically exposes the subject to the first link of a two-component chain (i.e., the standard or sample stimulus). A response to this stimulus produces the terminal link stimulus (i.e., the comparison stimulus). Matching to sample can be thought of as concomitant two-component chains acquired through forward chaining.

Maze learning can also be considered a behavioral

I wish to thank Coleman Paul for his contributions to this work and Nancy Myers for typing the final draft. Reprints may be obtained from R. Pisacreta, Department of Psychology, Ferris State College, Big Rapids, Michigan 49307. chain acquired via forward chaining. The rat is typically placed at the beginning of the maze and is always exposed to the sequence of choice points in a forward chaining direction. That is, he is not placed at the last choice point first and then over time exposed to the last two choice points, the last three, four, and so on, until a trial is reached that places him at the beginning of the maze.

Recent evidence can be cited to question the superiority of a backward chaining procedure over a forward chaining procedure. Straub, Seidenberg, Bever, and Terrace (1979) demonstrated that pigeons would acquire a serial learning task only when forward chaining was used. Boren (1969) and Boren and Devine (1968) taught monkeys four-component chains using a forward chaining procedure. Sidman and Rosenberger (1967) taught monkeys a serial learning task via forward chaining. Redd, Sidman, and Fletcher (1974) demonstrated that monkeys could learn a nine-component chain when the links were added in a forward direction (i.e., the last links to be acquired were introduced last). Keehn (1967) required rats to press two levers in sequence (a two-component chain) in order to escape and avoid electric shock. Both animals trained with forward chaining successfully established the chain. Only one of the two rats trained with a backward chaining procedure succeeded in acquiring the chain.

Finally, Weiss (1978), employing a six-key intelligence panel, taught college students four six-link response chains. Each subject learned two chains via backward chaining and two chains through forward chaining. The results indicated that all 10 subjects made fewer total errors acquiring two chains via forward chaining relative to the cumulative errors produced while acquiring two other chains through backward chaining. Weiss concluded that "each response in a forward chain procedure is directly reinforced, whereas only the terminal response 
is directly reinforced in a backward chain, leads to the prediction that forward chaining would be superior for the acquisition of a chain" (Weiss, 1978, p. 259).

The purpose of the present study was to compare the forward and backward chaining procedures employing pigeons as the subjects.

\section{METHOD}

\section{Subjects}

Four naive White Carneaux pigeons, maintained at $80 \% \pm 15 \mathrm{~g}$ of their free-feeding weights, were employed.

\section{Apparatus}

The apparatus was a $35 \times 35 \times 27 \mathrm{~cm}$ operant conditioning chamber enclosed in a sound-attenuating hull. A $20 \times 25 \mathrm{~cm}$ piece of one-way glass mounted on the door of the outer hull enabled observation of the entire experimental area.

The intelligence panel, $37 \times 35 \mathrm{~cm}$, employed standard dimensions and commercial components. Each key was $2.7 \mathrm{~cm}$ in diameter (BRS/LVE Model 121-16). Stimuli were nine response keys arranged in a 3 by 3 matrix. For reference, the keys were numbered from upper left (1) across to lower right (9). The stimuli were projected onto the keys by Industrial Electronics Engineers in-line projectors (Model 1813-44). The operating-force requirement of each key was approximately $.16 \mathrm{~N}$. The horizontal and vertical distances between the keys were 8.1 and $6.4 \mathrm{~cm}$, respectively, center to center. A 6 by 6 feeder aperture was centered on the wall $10 \mathrm{~cm}$ above the floor.

The feeder (BRS/LVE Model 114-10) provided $3 \mathrm{sec}$ access to grain. The houselight, a GE 1820 lamp, provided light before and after daily experimental sessions. During sessions, illumination was provided only by the nine in-line projectors. White noise delivered through a speaker and a ventilation fan masked extraneous noise. An E and L Instruments MMD-1 computer recorded data and controlled experimental events.

\section{Procedure}

Each subject was required to learn two four-link chains, one by forward chaining and the other through backward chaining. Four colors served as the discriminative stimuli $\left(\mathrm{SD}_{\mathrm{S}}\right)$ in the first chain learned, and four forms served as the $\mathrm{SD}_{\mathrm{S}}$ in the second chain. Figure 1 shows the chains employed.

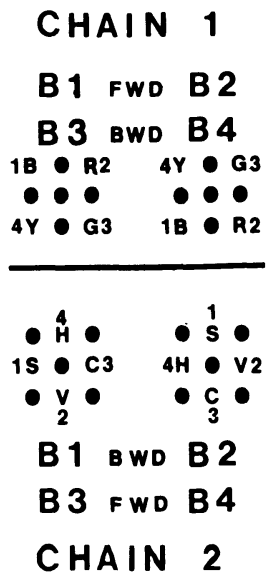

Figure 1. The chains employed. Each letter-number pair represents the correct key (number) to peck in the presence of a particular stimulus (letter). Filled circles represent keys that produced time-outs. The stimuli employed included: blue $(B)$, red (R), green (G), yellow (Y), horizontal line (H), square (S), circle (C), and vertical line (V).
Table 1

Summary of Chains Presented for One Session

\begin{tabular}{lcc}
\hline & Subjects B2 and B4 & Subjects B1 and B3 \\
\hline Original Chain & S2, V6, C8, H4 & S4, V8, C6, H2 \\
Scramble 1 & V6, C8, H4, S2 & V9, C6, H2, S4 \\
Scramble 2 & C8, H4, S2, V6 & C6, H2, S4, V8 \\
Scramble 3 & H4, S2, V6, C8 & H2, S4, V9, C6 \\
\hline
\end{tabular}

Note-Each letter-number pair represents the correct key (number) to peck in the presence of a particular stimulus (letter). $S=$ square, $V=$ vertical line, $C=$ circle, and $H=$ horizontal line.

The subjects were equally divided into two groups: (1) forward chain first (Subjects B1 and B2) and (2) backward chain first (Subjects B3 and B4). The four corner keys $(1,3,7,9)$ were employed as the first chain. For all subjects, the terminal stimulus sequence was blue (B), red (R), green $(G)$, and yellow (Y).

Initially, all subjects were magazine trained and shaped to the single blue (for Subjects B1 and B2) or yellow (for Subjects B3 and B4) response key that served as the first link to be learned in their respective chains. Each subject was then given 20 additional sessions of continuous reinforcement (CRF) for pecking this key. Each session ended after 50 reinforcements. On the 21 st session, the keys 1-9 inclusive presented the blue or yellow stimulus. The reinforcement contingency was the same as before: CRF for pecking the correct key. A response on any other (incorrect) key produced a 3-sec time-out (TO). During TO, all stimuli were eliminated and the chamber was dark. The criteria for adding an additional link was less than three errors for each of three consecutive sessions.

Figure 1 shows that the key sequence was varied across subjects in order to counterbalance for the direction of the chain (clockwise vs. counterclockwise), the type of chain (forward vs. backward), and the sequence (emitting the chain from the lower keys to the upper keys and vice versa). For example, Figure 1 indicates that for the first chain, Subject B1 learned a forward (FWD) chain in a clockwise direction, which was emitted from the upper left key to the lower left key. The final sequence was: In the presence of blue, peck Key 1 ; in the presence of red, peck Key 3 ; in the presence of green, peck Key 9; in the presence of yellow, a response on Key 7 produced reinforcement, a 3 -sec intertrial interval (ITI), and the next trial. For the second chain, four forms were employed as the $S^{D_{s}}$ : a vertical line $(\mathrm{V})$, a circle $(\mathrm{C})$, a horizontal line $(\mathrm{H})$, and a square (S). Respone Keys $2,4,6$, and 8 were the designated chain sequence. For example, Subject B1 learned a backward (BWD) chain in a counterclock wise direction, which was emitted from the center left key (4) to the upper center key (2). Similar counterbalancing was provided for the other subjects (see Figure 1).

It is possible for the birds to learn a position sequence, that is, to peck four keys in a particular order without coming under control of the four $\mathrm{S}^{\mathrm{D}_{S}}$ provided. In order to test for this possibility, the four links of the second chain learned were presented to the subjects with their order rearranged (scrambled) during one session. If responding was under the control of the $S^{D}$, then each bird should have emitted few errors regardless of the order in which the four links were presented. Table 1 provides the sequences used.

In order to assess the response strength of the chains, extinction was introduced for one session. During this session, completion of a chain produced only the first-link $S^{D}$ of the next chain. The session ended when the subject had not responded for $1 \mathrm{~h}$.

\section{RESULTS}

Figure 2 illustrates the cumulative number of errors emitted by each subject and the number of sessions 


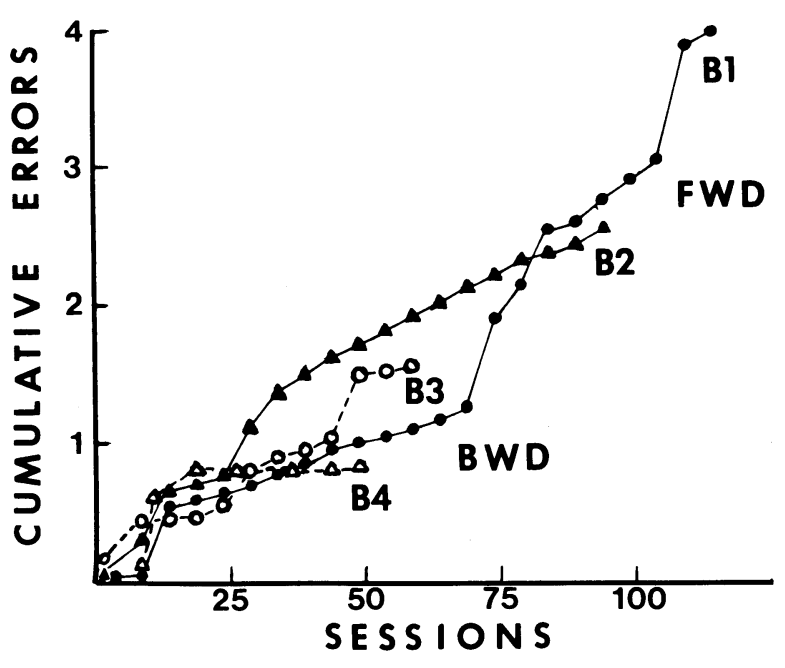

Figure 2. Cumulative errors (in thousands) made during the acquisition of the first chain. Each data point represents the sum of five sessions.

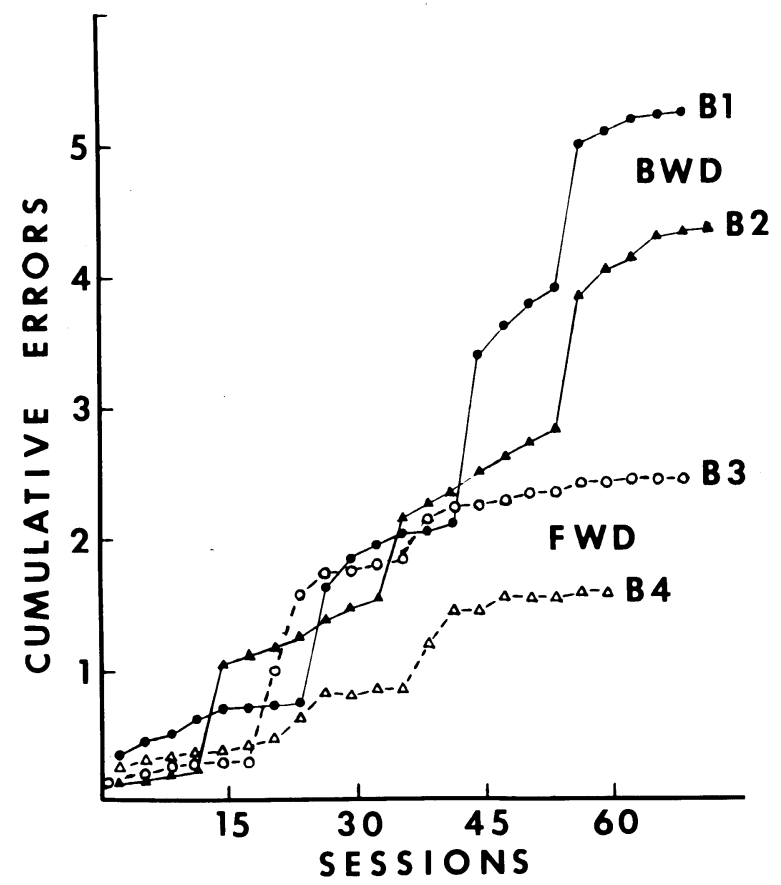

Figure 3. Cumulative errors (in thousands) made while acquiring the second chain. Each data point represents the sum of three sessions.

required for each bird to acquire the first chain. Figure 2 shows that both backward chaining birds, B3 and B4, emitted fewer errors and required fewer sessions to acquire the first chain than did the forward chaining birds. The majority of errors were produced during the first three sessions a new link was introduced.

Figure 3 shows the cumulative errors emitted by each bird while acquiring the second chain. Figure 3 shows that both forward chaining birds, B3 and B4, emitted fewer errors and required fewer sessions to acquire the second chain than did the backward chaining birds, B1 and B2. There are two ways to interpret these results: (1) Pigeons B3 and B4 are better at acquiring chains than are B1 and B2 regardless of the procedure used, forward or backward, and (2) the pigeons make fewer errors acquiring the first chain regardless of the procedure.

A comparison of Figures 2 and 3 reveals that pigeons can learn heterogeneous chains via forward chaining as well as they learn respone sequences through backward chaining. All subjects produced more errors while acquiring the second chain than they did while acquiring the first. In terms of errors, this may indicate that acquisition of the first chain interferes with the acquisition of the second chain. Each bird did, however, require fewer sessions to acquire the second chain. Finally, each bird required fewer sessions to acquire the backward chain relative to the forward chain.

The errors produced while acquiring a chain followed the same pattern for each bird: (1) persevering on the key that used to function as the first $S^{D},(2)$ generalized responding across the keys until the correct key is pecked, (3) responding on keys that are adjacent to the correct key, and (4) reduction in these responses to TO keys, resulting in each bird's satisfying the chain acquisition criteria. The introduction of each new link produced a disruption in the established links. The birds often pecked the correct key, which produced the $S^{\mathbf{D}}$ for the next link. However, they often pecked the same key again, producing a TO before moving on to the correct key for that link. There was a direct correlation between the number of links required and the number of errors produced (i.e., more links, more errors).

Both birds produced more errors, link for link, acquiring the second chain than they did acquiring the first chain. Responses to Key 5 increased for both birds, although pecks to this key always produced a TO. Despite the use of forms as $S^{D}$ and former TO keys as $S^{D}$ keys, the distributions of errors during the second chain were similar to those produced while acquiring the first chain.

Table 2 presents the total errors produced in which the scrambled chains were presented. Table 2 indicates that each subject's behavior was under the control of the stimuli presented rather than a position sequence

Table 2

Total Errors Produced With Scrambled Chain Lines

\begin{tabular}{|c|c|c|c|c|c|c|c|c|}
\hline & \multicolumn{8}{|c|}{ Subject } \\
\hline & \multicolumn{4}{|c|}{ Run 1} & \multicolumn{4}{|c|}{ Run 2} \\
\hline & B1 & B2 & B3 & B4 & B1 & B2 & B3 & B4 \\
\hline Scramble 1 & 4 & 0 & 5 & 4 & 7 & 2 & 1 & 1 \\
\hline Scramble 2 & 0 & 7 & 5 & 3 & 6 & 8 & 2 & 4 \\
\hline Scramble 3 & 4 & 2 & 5 & 2 & 1 & 4 & 4 & 2 \\
\hline Twice & 1 & 0 & 0 & 0 & 1 & 0 & 0 & 0 \\
\hline Twice & 1 & 1 & 2 & 0 & 0 & 2 & 1 & 0 \\
\hline Total & 10 & 10 & 15 & 9 & 15 & 16 & 8 & 7 \\
\hline
\end{tabular}

Note-Each altered chain was presented twice, Run 1 and Run 2. "Twice" refers to a trial in which the original chain had to be emitted two times (eight responses). 
Table 3

Extinction Data

\begin{tabular}{ccccc}
\hline Subject & $\begin{array}{c}\text { Chain } \\
\text { Direction }\end{array}$ & $\begin{array}{c}\text { Completed } \\
\text { Chains }\end{array}$ & $\begin{array}{c}\text { Total } \\
\text { Errors }\end{array}$ & $\begin{array}{c}\text { Chain of } \\
\text { First Error }\end{array}$ \\
\hline B1 & B & 215 & 520 & 2 \\
B2 & B & 303 & 676 & 6 \\
B3 & F & 261 & 392 & 15 \\
B4 & F & 372 & 866 & 13 \\
\hline
\end{tabular}

Note-Chain direction is indicated as backward (B) or forward (F).

emitted regardless of stimulus conditions. The majority of the errors were produced by pecking the correct key twice rather than by responding on an incorrect key. This result may indicate that in the present study we are dealing with four discriminations that are emitted as an experimenter-designated chain. Emission of the responses in a particular sequence does not necessarily contribute to the overall performance. Specifically, the bird's physical position to the keys and propriocentive information during the emission of the links of a sequence have little control as $\mathrm{S}^{\mathrm{D}} \mathrm{s}$.

Finally, Table 3 presents the extinction data produced by each subject. Birds B1 and B2 (backward chaining) produced their first errors earlier than did the forward chaining birds. A closer analysis of the data showed that the backward chaining birds yielded more errors in the first link of each chain, whereas the forward chaining subjects emitted the majority of their errors in the last link of each chain. Once a bird responded to the first link of a chain, it quickly emitted the rest of the chain. The latency to respond to the first link of a chain increased over trials until each bird satisfied the extinction criterion of not responding for $1 \mathrm{~h}$.

\section{DISCUSSION}

The results indicate that neither the forward nor the backward chaining procedure proved to be consistently superior in establishing a four-link chain. There are procedural factors involved in the present experiment that deserve further comment.

When a novel link is introduced in a forward chain, it is produced by a response to the former last link. The subject is then in the presence of a novel stimulus. Weiss (1978) points out that, at this time, the subject is directly reinforced for responding to the new correct last link.

In the backward chaining procedure, the subject begins the trial in the presence of the novel, new-link stimulus. A correct response replaces the novel stimulus with a familiar stimulus, the former first-link $\mathrm{SD}^{\mathrm{D}}$. The bird has a tendency to respond to the nearest key presenting this stimulus. Since the bird is still proximal to the key that produced this stimulus, a second response on this key is counted as an error. These two factors would seem to predict fewer errors with a forward chaining procedure. The data, however, do not support this position.

Due to the spatial separation of the keys, the procedure is defined as a heterogeneous chain. The question remains as to whether a four-link chain employing pigeons and pecking keys is as heterogeneous as a chain that would utilize four different operants.

Unlike conventional chaining demonstrations, the Weiss (1978) experiment and this study do not employ shaping of new links to be acquired. In both studies, new links were acquired with generalized responding on a trial-and-error basis, rather than secondary or primary reinforcement of successive approximations to the new correct reponse.

In the present study, the subjects could have established incorrect responses followed by correct responses as a superstitious chain when new links were added. All subjects did initially respond on incorrect keys that were spatially between two consecutive correct responses. These errors and double reponses on correct keys account for the majority of the errors. Over trials, these incorrect responses would diminish due to the TOs they produce. At this point, the subject would emit only the correct chain. These superstitious acquisition errors may account for the failure of one chaining procedure to prove to be more effective than the others. Redd et al. (1974) and Schwartz (1980) both have provided demonstrations of the acquisition of these "stereotyped errors."

More research on the transition phenomenon called chaining is needed. Studying how organisms acquire complex sequences may reveal biological and structural universals that govern the formation of complex behavior in animals and humans.

\section{REFERENCES}

Boren, J. J. Some variables affecting the superstitious chaining of responses. Journal of the Experimental Analysis of Behavior, $1969,12,959-969$.

Boren, J. J., \& Devine, D. D. The repeated acquisition of behavioral chains. Journal of the Experimental Analysis of Behavior, 1968, 11, 651-660.

KEEHN, J. D. Is bar-holding with negative reinforcement preparatory or perseverative? Journal of the Experimental Analysis of Behavior, 1967, 10, 461-465.

Millenson, J. R. Principles of behavioral analysis. New York: Macmillan, 1967.

Pierrel, R., \& Sherman, J. G. Train your pet the Barnabus way. Brown Alumni Monthly, February 1963, pp. 8-14.

Rachlin, H. Behavior and learning. San Francisco: Freeman, 1976.

Redd, W. H., Sidman, M., \& Fletcher, G. F. Timeout as a reinforcer for errors in a serial position task. Journal of the Experimental Analysis of Behavior, 1974, 21, 3-17.

Schwartz, B. Development of complex, stereotyped behavior in pigeons. Journal of the Experimental Analysis of Behavior, $1980,33,153-166$.

Sidman, M., \& Rosenberger, P. B. Several methods for teaching serial position sequences to monkeys. Journal of the Experimental Analysis of Behavior, 1967, 10, 467-478.

Skinner, B. F. The behavior of organisms: An experimental analysis. New York: Appleton-Century-Crofts, 1938.

Straub, R. O., Seidenberg, M. S., Bever, T. G., \& Terrace, H. S. Serial learning in the pigeon. Journal of the Experimental Analysis of Behavior, 1979, 32, 137-148.

WEISs, K. M. A comparison of forward and backward procedures for the acquisition of response chains in humans. Journal of the Experimental Analysis of Behavior, 1978, 29, 255-259.

Williams, J. L. Operant learning: Procedures for changing behavior. Monterey, Calif: Brooks/Cole, 1973.

(Received for publication July 20, 1982.) 